\title{
Autologous mitochondrial transplantation for dysfunction after ischemia-reperfusion injury
}

\author{
Sitaram M. Emani, MD, ${ }^{a}$ Breanna L. Piekarski, RN, BSN, ${ }^{a}$ David Harrild, MD, ${ }^{b}$ Pedro J. del Nido, MD, ${ }^{a}$ \\ and James D. McCully, PhD, ${ }^{a}$ Boston, Mass
}

From the Departments of a Cardiac Surgery and ${ }^{b}$ Cardiology, Boston Children's Hospital, Boston, Mass.

Supported by The Richard A. and Susan F. Smith Foundation, President's Innovation Award, Boston Children's Hospital; Michael B. Klein and Family; the Sidman Family Foundation; the Bulens/Capozzi Foundation; and the Kenneth C. Griffin Charitable Research Fund.

The authors attest that they had full freedom to explore the data and analyze the results independently of any sponsor and that they had sole authority to make the final decision to submit the material for publication.

Disclosures: J.D.M. and P.J.dN. have patents pending for the isolation and usage of mitochondria have patents pending for the isolation and usage of mitochondria. All other authors have nothing to disclose with regard to commercial support.

Received for publication Oct 12, 2016; revisions received Dec 23, 2016; accepted for publication Feb 8, 2017; available ahead of print March 7, 2017.

Address for reprints: Sitaram M. Emani, MD, Boston Children's Hospital, 300 Longwood Ave, Bader 273, Boston, MA 02115 (E-mail: Sitaram.Emani@cardio.chboston.org).

J Thorac Cardiovasc Surg 2017;154:286-9

$0022-5223 / \$ 36.00$

Copyright (C) 2017 by The American Association for Thoracic Surgery

http://dx.doi.org/10.1016/j.jtcvs.2017.02.018

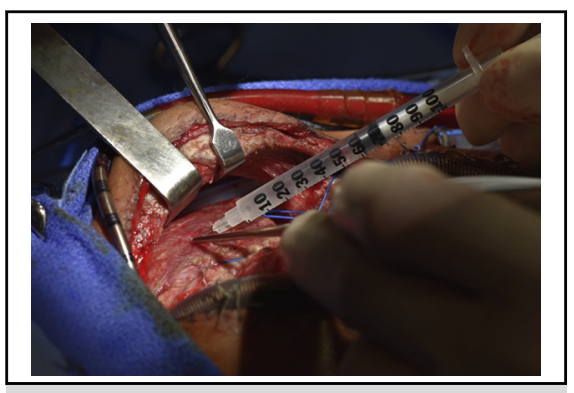

Intramyocardial injection of autologous mitochondria with a tuberculin syringe.

\section{Central Message}

Healthy autologous mitochondria harvested from nonischemic skeletal muscle can be safely injected into damaged myocardium after ischemic injury for improvement in ventricular function.

See Editorial Commentary page 290.

See Editorial page 284.
Current treatments for pediatric patients who have had myocardial ischemia-reperfusion injury include inotropic and mechanical circulatory support. Recovery of myocardial function after extracorporeal membrane oxygenation (ECMO) support is inconsistent, as reflected by a $40 \%$ failure to separate from ECMO. ${ }^{1}$ Mitochondrial damage and dysfunction contribute significantly to the myocardial dysfunction in such patients with ischemia-reperfusion injury. $^{2}$ A novel strategy to repair and replenish damaged mitochondria, termed mitochondrial autotransplantation, has been developed in which healthy autologous mitochondria harvested from nonischemic skeletal muscle are transplanted into injured myocardium. ${ }^{3}$ Previous reports have demonstrated that transplanted mitochondria restore mitochondrial function and viability and improve postischemic myocardial function by internal and extracellular mechanisms that include high-energy synthesis, transcriptomic and proteomic alteration, and DNA repair. ${ }^{2-5}$

\section{MATERIALS AND METHODS}

Pediatric patients who required central ECMO support for ischemiareperfusion-associated myocardial dysfunction after cardiac surgical procedure were eligible for mitochondrial autotransplantation. Patients were included if they had a myocardial ischemic event after cardiac surgery that was not ameliorated by surgical intervention and ECMO support. Patients were excluded if they underwent ECMO cannulation through peripheral vessels (cervical or femoral), because access for myocardial injections is not possible with this approach.
Mitochondrial harvest and isolation can be performed within 20 to $30 \mathrm{mi}-$ nutes during the same procedure and involves minimal manipulation of muscle tissue. Review of the proposed therapy was provided by 2 independent physicians who were not involved with the patient's care, and families were extensively counseled regarding the potential risks of the procedure. The treatment was provided under an Innovative Therapies protocol developed by the Boston Children's Hospital's institutional review board.

In all patients, the mediastinum was accessed and epicardial echocardiography was performed to identify regions of myocardial akinesis or hypokinesis. A $6 \times 6-\mathrm{mm}$ piece of healthy rectus abdominis muscle was harvested from the inferior aspect of the field by sharp dissection (Figure 1, A). Autologous mitochondria $\left(1 \times 10^{8} \pm 1 \times 10^{5}\right)$ were isolated under sterile conditions and suspended in $1 \mathrm{~mL}$ respiration buffer. $^{2,5}$ Ten $100-\mu \mathrm{L}$ injections containing $1 \times 10^{7} \pm 1 \times 10^{4}$ mitochondria each were delivered by direct injection with a $1-\mathrm{mL}$ tuberculin syringe (28-gauge needle) to the myocardium affected by ischemia-reperfusion, as identified by epicardial echocardiography (Figure 1, B). Epicardial echocardiography was performed at the conclusion of the procedure to assess the presence of myocardial hematoma related to injections.

Echocardiograms were read by a blinded reviewer for both global and regional dysfunction segments during the time reported (Videos 1 and 2).

\section{RESULTS}

The characteristics and outcomes (mortality and global cardiac function and regional hypokinesis segments) of the patients who underwent mitochondrial autotransplantation are 

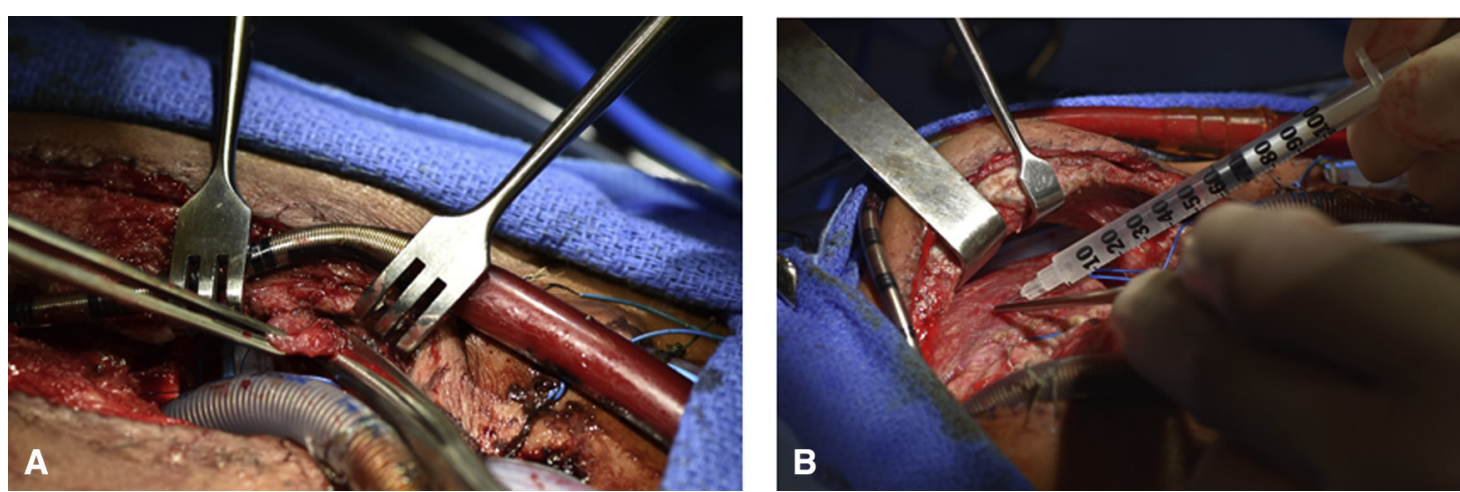

FIGURE 1. A, Biopsy of nonischemic skeletal muscle. B, Injection of autologous mitochondria into the myocardium with an insulin syringe.

described in Table 1. None of the patients had arrhythmias or bleeding related to epicardial injections. Of the 5 subjects, 4 demonstrated improvement in ventricular function and were successfully separated from ECMO support.

\section{DISCUSSION}

This report describes the use of mitochondrial autotransplantation for myocardial recovery in pediatric patients who require ECMO support as a result of ischemia-reperfusion injury. Patients did not have adverse short-term complications related to mitochondrial injection (arrhythmia, intramyocardial hematoma, or scarring), and all demonstrated improvement in ventricular function within several days after treatment. Mitochondrial therapy is most advantageous if delivered as soon after ischemic injury as possible, as evidenced by studies in animal models. The patients in this series, however, were selected because they showed no recovery of myocardial function despite 1 to 2 days of ECMO support, and spontaneous recovery of ventricular function did not seem likely. Future studies investigating the optimal timing of therapy are necessary. It is possible that ventricular function might

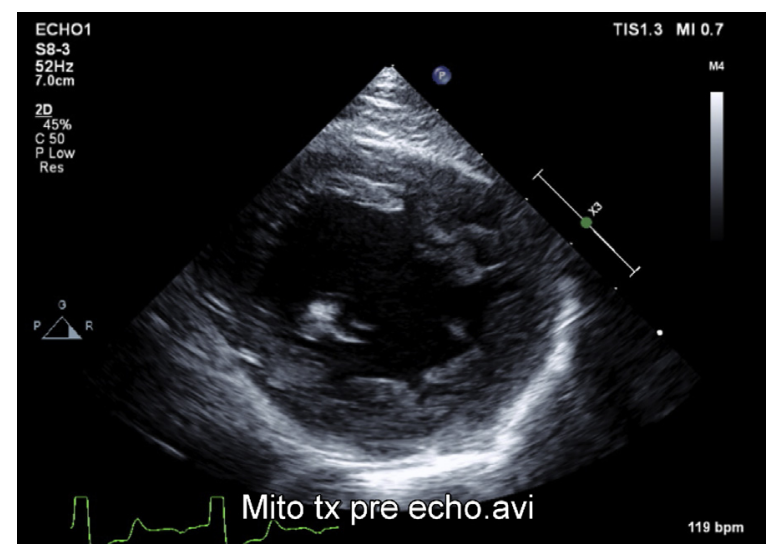

VIDEO 1. Ventricular function according to echocardiography of patient 2 before mitochondrial autotransplantation. Video available at: http://www. jtcvsonline.org/article/S0022-5223(17)30258-1/addons. have improved without mitochondrial autotransplantation, and a randomized clinical trial is therefore necessary to demonstrate the efficacy of the strategy.

The dose of mitochondria and the method of delivery in this study were based on previous animal experience and extrapolated to human patient cardiac mass. ${ }^{2}$ Future dose escalation studies are necessary to determine the optimal dose of mitochondria necessary in human patients. Although epicardial injection was used in this study, alternative delivery methods, including transcoronary delivery, are currently under investigation.

There was no detectable difference between preinjection and postinjection markers of systemic inflammatory response syndrome (as evidenced by stable respiratory and renal status), in agreement with animal study data. ${ }^{2}$ Autopsy of patient 1 revealed no signs of inflammation or rejection at the sites of injection, and white blood cell counts had no clinically relevant change.

\section{CONCLUSIONS}

These cases demonstrate the first clinical application of a novel technique of mitochondrial autotransplantation that may be useful for patients with ischemia-reperfusion injury.

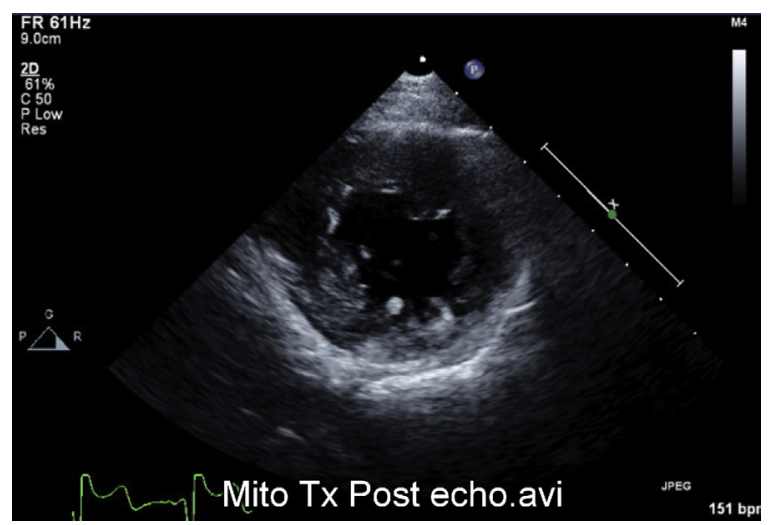

VIDEO 2. Ventricular function according to echocardiography of patient 2 after mitochondrial autotransplantation. Video available at: http://www. jtcvsonline.org/article/S0022-5223(17)30258-1/addons. 
TABLE 1. Patient characteristics and outcomes

\begin{tabular}{|c|c|c|c|c|c|}
\hline Characteristic & Case 1 & Case 2 & Case 3 & Case 4 & Case 5 \\
\hline Sex & Male & Female & Female & Female & Male \\
\hline Age & $4 \mathrm{~d}$ & $2 y$ & $6 \mathrm{~d}$ & $6 \mathrm{mo}$ & $25 \mathrm{~d}$ \\
\hline Diagnosis & D-TGA & Tricuspid atresia 1B & HLHS & LVOTO & D-TGA \\
\hline Surgical repair & ASO & Fontan & $\begin{array}{l}\text { Stage } 1 \text { Norwood and } \\
\text { RmBTS }\end{array}$ & Ross procedure & ASO \\
\hline $\begin{array}{l}\text { Cause of ischemic } \\
\text { injury }\end{array}$ & $\begin{array}{l}\text { Occlusion of } \\
\text { reimplanted LCA }\end{array}$ & $\begin{array}{l}\text { Occlusion of LCA s/t } \\
\text { suture at LA } \\
\text { appendage }\end{array}$ & $\begin{array}{l}\text { External compression } \\
\text { of DKS and RCA } \\
\text { by hemostatic agent }\end{array}$ & $\begin{array}{l}\text { Small and tortuous } \\
\text { LCA }\end{array}$ & $\begin{array}{l}\text { LV distention and } \\
\text { subendocardial } \\
\text { ischemia }\end{array}$ \\
\hline $\begin{array}{l}\text { Ischemic injury } \\
\text { intervention }\end{array}$ & $\begin{array}{l}\text { Revision of } \\
\text { aortocoronary } \\
\text { anastomosis }\end{array}$ & $\begin{array}{l}\text { Suture removal with } \\
\text { successful } \\
\text { restoration of flow }\end{array}$ & $\begin{array}{l}\text { Removal of } \\
\text { hemostatic agent } \\
\text { and mediastinal } \\
\text { compressing } \\
\text { thrombus }\end{array}$ & $\begin{array}{l}\text { Removal of } \\
\text { hemostatic agent } \\
\text { and LCA } \\
\text { mobilization }\end{array}$ & LA vent \\
\hline $\begin{array}{l}\text { Duration between } \\
\text { ECMO cannulation } \\
\text { and treatment }\end{array}$ & $15 \mathrm{~d}$ & $4 \mathrm{~d}$ & $2 \mathrm{~d}$ & $3 \mathrm{~d}$ & $4 \mathrm{~d}$ \\
\hline $\begin{array}{l}\text { Time from treatment } \\
\text { to decannulation }\end{array}$ & NA & $3 d$ & $6 \mathrm{~d}$ & $3 \mathrm{~d}$ & $4 \mathrm{~d}$ \\
\hline \multicolumn{6}{|l|}{$\begin{array}{l}\text { Cardiac segmentation } \\
\text { schema for } \\
\text { subsequent data }\end{array}$} \\
\hline Injection site & Segments 1,2 & Segment 3 & Segments $4,5,6$ & Segments 2,3 & Segments $1,2,3$ \\
\hline $\begin{array}{l}\text { Ventricular function } \\
\text { before treatment, by } \\
\text { echocardiogram }\end{array}$ & $\begin{array}{l}\text { Global: moderate LV } \\
\text { systolic } \\
\text { dysfunction; } \\
\text { Regional } \\
\text { hypokinesia: } \\
\text { segments } 1,2,3\end{array}$ & $\begin{array}{l}\text { Global: severe LV } \\
\text { systolic } \\
\text { dysfunction; } \\
\text { Regional } \\
\text { hypokinesia: } \\
\text { segments 3, } 4\end{array}$ & $\begin{array}{l}\text { Global: severe RV } \\
\text { systolic } \\
\text { dysfunction; } \\
\text { Regional } \\
\text { hypokinesia: } \\
\text { segments 4, 5, } 6\end{array}$ & $\begin{array}{l}\text { Global: moderate- } \\
\text { severe LV systolic } \\
\text { dysfunction; } \\
\text { Regional } \\
\text { hypokinesia: } \\
\text { segments } 2,3,4\end{array}$ & $\begin{array}{l}\text { Global: Severe LV } \\
\text { systolic } \\
\text { dysfunction; } \\
\text { Regional } \\
\text { hypokinesia: } \\
\text { segments 1, 2, } 3\end{array}$ \\
\hline $\begin{array}{l}\text { Ventricular function } \\
24 \mathrm{~h} \text { after treatment, } \\
\text { by echocardiogram }\end{array}$ & $\begin{array}{l}\text { Global: mild LV } \\
\text { systolic } \\
\text { dysfunction; } \\
\text { Regional } \\
\text { hypokinesia: } \\
\text { segments 1, 2, } 3\end{array}$ & $\begin{array}{l}\text { Global: moderate LV } \\
\text { systolic } \\
\text { dysfunction; } \\
\text { Regional } \\
\text { hypokinesia: } \\
\text { segments 3, } 4\end{array}$ & NA & $\begin{array}{l}\text { Global: severe LV } \\
\text { systolic } \\
\text { dysfunction; } \\
\text { Regional } \\
\text { hypokinesia: } \\
\text { segments 2, 3, } 4\end{array}$ & $\begin{array}{l}\text { Global: mild LV } \\
\text { systolic } \\
\text { dysfunction; } \\
\text { Regional } \\
\text { hypokinesia: } \\
\text { segment } 2\end{array}$ \\
\hline $\begin{array}{l}\text { Ventricular function } \\
48 \mathrm{~h} \text { after treatment, } \\
\text { by echocardiogram }\end{array}$ & $\begin{array}{l}\text { Global: Mild LV } \\
\text { systolic } \\
\text { dysfunction; } \\
\text { Regional } \\
\text { hypokinesia: } \\
\text { segment } 2\end{array}$ & NA & NA & $\begin{array}{l}\text { Global: Mild- } \\
\text { moderate LV } \\
\text { systolic } \\
\text { dysfunction; } \\
\text { Regional } \\
\text { hypokinesia: } \\
\text { segments 2, } 3\end{array}$ & $\begin{array}{l}\text { Global: Mild LV } \\
\text { systolic } \\
\text { dysfunction; } \\
\text { Regional } \\
\text { hypokinesia: } \\
\text { segments 2, } 3\end{array}$ \\
\hline $\begin{array}{l}\text { Ventricular function 4- } \\
6 \mathrm{~d} \text { after treatment, } \\
\text { by echocardiogram }\end{array}$ & $\begin{array}{l}\text { Global: mild LV } \\
\text { systolic } \\
\text { dysfunction; } \\
\text { Regional } \\
\text { hypokinesia: } \\
\text { segment } 2\end{array}$ & $\begin{array}{l}\text { Global: mild LV } \\
\text { systolic } \\
\text { dysfunction; } \\
\text { Regional } \\
\text { hypokinesia: none }\end{array}$ & $\begin{array}{l}\text { Global: normal RV } \\
\text { systolic function; } \\
\text { Regional } \\
\text { hypokinesia: none }\end{array}$ & $\begin{array}{l}\text { Global: mild to } \\
\text { moderate LV } \\
\text { systolic } \\
\text { dysfunction; } \\
\text { Regional } \\
\text { hypokinesia: } \\
\text { segments 2, } 3\end{array}$ & $\begin{array}{l}\text { Global: borderline- } \\
\text { mild LV systolic } \\
\text { dysfunction; } \\
\text { Regional } \\
\text { hypokinesia: none }\end{array}$ \\
\hline
\end{tabular}


TABLE 1. Continued

\begin{tabular}{|c|c|c|c|c|c|}
\hline Characteristic & Case 1 & Case 2 & Case 3 & Case 4 & Case 5 \\
\hline $\begin{array}{l}\text { Ventricular function } \\
10 \mathrm{~d} \text { after treatment, } \\
\text { by echocardiogram }\end{array}$ & NA & $\begin{array}{l}\text { Global: Mild LV } \\
\text { systolic } \\
\text { dysfunction; } \\
\text { regional } \\
\text { hypokinesia: none }\end{array}$ & NA & $\begin{array}{l}\text { Global: Normal LV } \\
\text { systolic function; } \\
\text { regional } \\
\text { hypokinesia: none }\end{array}$ & $\begin{array}{l}\text { Global: Normal LV } \\
\text { systolic function; } \\
\text { regional } \\
\text { hypokinesia: none }\end{array}$ \\
\hline Mortality & Dead & Alive & Dead & Alive & Alive \\
\hline Current status & $\begin{array}{l}\text { Despite recovery of } \\
\text { myocardial } \\
\text { function, patient did } \\
\text { not tolerate } \\
\text { decannulation due } \\
\text { to persistent } \\
\text { pulmonary, renal, } \\
\text { and hepatic } \\
\text { insufficiency. }\end{array}$ & $\begin{array}{l}\text { Patient discharged on } \\
\text { POD 38; } \\
\text { echocardiogram } \\
403 \text { d after therapy } \\
\text { showed global } \\
\text { moderate } \\
\text { dysfunction. }\end{array}$ & $\begin{array}{l}\text { On POD 30, patient } \\
\text { had mild LV } \\
\text { dysfunction; patient } \\
\text { ultimately died of } \\
\text { respiratory } \\
\text { insufficiency after } \\
\text { BDG at } 4 \text { mo of age. }\end{array}$ & $\begin{array}{l}\text { Patient discharged on } \\
\text { POD 52; } \\
\text { echocardiogram } \\
119 \text { d after therapy } \\
\text { showed global } \\
\text { borderline } \\
\text { dysfunction. }\end{array}$ & $\begin{array}{l}\text { Patient discharged on } \\
\text { POD 30; } \\
\text { echocardiogram } \\
34 \text { d after therapy } \\
\text { showed global mild } \\
\text { dysfunction. }\end{array}$ \\
\hline
\end{tabular}

$\overline{D-T G A}$, Dextrotransposition of the great arteries; HLHS, hypoplastic left heart syndrome; LVOTO, left ventricular outflow tract obstruction; ASO, arterial switch operation; $R m B T S$, right modified Blalock-Taussig Shunt; $L C A$, left coronary artery; $s / t$, secondary to; $L A$, left atrium; $D K S$, Damus-Kaye-Stansel procedure; $R C A$, right coronary artery; $E C M O$, extracorporeal membrane oxygenation; $N A$, not applicable; $L V$, left ventricle; $R V$, right ventricle; $P O D$, postoperative day; $B D G$, bidirectional Glenn shunt.

Prospective clinical trials are warranted to assess safety, efficacy, and optimal dosing of the therapy.

\section{References}

1. Nasr VG, Faraoni D, DiNardo JA, Thiagarajan RR. Association of hospital structure and complications with mortality after pediatric extracorporeal membrane oxygenation. Pediatr Crit Care Med. 2016;17:684-91.

2. Masuzawa A, Black KM, Pacak CA, Ericsson M, Barnett RJ, Drumm C, et al. Transplantation of autologously-derived mitochondria protects the heart from ischemia-reperfusion injury. Am J Physiol Heart Circ Physiol. 2013;304: H966-82.

3. McCully JD, Levitsky S, del Nido PJ, Cowan DB. Mitochondrial transplantation for therapeutic use. Clin Transl Med. 2016;5:16.

4. Lesnefsky E, Hoppel C. Ischemia-reperfusion injury in the aged heart: role of mitochondria. Arch Biochem Biophys. 2003;420:287-97.

5. Kaza AK, Wamala I, Friehs I, Kuebler JD, Rathod RH, Berra I, et al. Myocardial rescue with autologous mitochondrial transplantation in a porcine model of ischemia/reperfusion. J Thorac Cardiovasc Surg. November 15, 2016 [Epub ahead of print]. 